\title{
O método psicanalítico e suas extensões: escutando jovens em situação de vulnerabilidade social*1
}

\author{
Perla Klautau*2
}

Este artigo visa discutir o trabalho de construção de espaços de escuta para jovens em situação de vulnerabilidade social. Para tal, foram configuradas duas modalidades de intervenção, desenvolvidas no âmbito individual e grupal, nomeadas, respectivamente, papo reto e tá na roda. Propostas como estas permitem o avanço em direção a estratégias de construção de dispositivos alternativos ao setting clássico, estendendo as contribuições do método psicanalítico para a saúde, para a educação e para a cultura.

Palavras-chaves: Psicanálise em extensão, vulnerabilidade social, trauma, identificação

*1 Este trabalho foi elaborado no âmbito da Pesquisa "Extensões do método Psicanalítico e conexões interdisciplinares", financiada pela FAPERJ e desenvolvida no Laboratório de Estudos em Psicanálise e Conexões Interdisciplinares (LEPCI), situado na Universidade Veiga de Almeida (UVA).

*2 Universidade Veiga de Almeida - UVA (Rio de Janeiro, RJ, Br). 


\section{Introdução}

Trabalhar com jovens em situação de vulnerabilidade social exige a adoção de uma visão complexa capaz de articular a dimensão psíquica com o contexto social, político e econômico. Neste artigo, o uso do termo jovem faz referência a uma faixa etária expandida entre os 15 e os 24 anos de idade. Neste período, a maioria dos sujeitos já vivenciou as alterações corporais características da puberdade e, com isso, encontra-se diante do trabalho de construir um lugar capaz de abrigar o novo corpo e todas as mudanças subjetivas e sociais atreladas a essa transformação. A tarefa de descobrir e significar o mundo que adquire uma nova roupagem coloca o jovem diante do trabalho de elaborar as descobertas e transformações suscitadas não só pelas mudanças corporais mas, sobretudo, pelo encontro com o sexo, pela assunção dos próprios desejos, sonhos e projetos de vida (Klautau \& Faissol, 2015-2016).

A falta de um lugar para expressar as angústias desencadeadas pelas inúmeras tentativas de desvendamentos de desejos e sofrimentos envolvidos na tentativa de construção de um lugar no mundo adulto coloca os jovens em uma posição vulnerável. Eles precisam de suportes identificatórios para o atravessamento da transitoriedade característica das descobertas em curso, mas geralmente os que sempre funcionaram como base de apoio não são mais considerados parceiros adequados. Na maioria das vezes são concebidos como ultrapassados para ancorar as identificações provisórias típicas desse espaço de tempo permeado por incertezas e instabilidades. Assim, no período em que o jovem precisa encontrar formas de estabelecer uma mediação entre os desejos projetados sobre si e a assunção de seus próprios desejos, o olhar dos pais não funciona mais como fonte suficiente de reconhecimento.

A esse tipo de vulnerabilidade é possível acrescentar a interação de diversos aspectos de ordem social, cultural e econômica 


\section{ARTIGOS}

que conduzem os jovens a situações de riscos e incertezas em relação à inserção no mercado de trabalho, ao acesso a bens de consumo e, sobretudo, à conquista de condições para a realização de sonhos e projetos de vida. Esses fatores evidenciam que a juventude é um período povoado por vulnerabilidades. O plural foi utilizado com o intuito de ressaltar, pelo menos, três diferentes faces da situação de vulnerabilidade que se encontram intimamente relacionadas: econômica, social e subjetiva. Independentemente da face a ser examinada, vulnerabilidade traz consigo a ideia de insegurança, incerteza e exposição a riscos.

De acordo com suas análises da estrutura social, Castel (1999) defende a ideia de que vulnerabilidade pode ser entendida como uma zona de risco formada "por aqueles que estão ameaçados, instáveis, frágeis, correndo o risco de cair na marginalidade" (p. 20). Segundo o autor, a marginalidade é resultante de um processo de desligamento que inclui, de um lado, a relação com o trabalho e, de outro, a inserção relacional. Esses dois aspectos, quando acoplados, configuram três zonas: zona de integração (trabalho estável e forte inserção relacional), zona de vulnerabilidade (trabalho precário e fragilidade dos apoios relacionais) e zona de desfiliação (ausência de trabalho e isolamento relacional). Isso faz com que a zona de vulnerabilidade ocupe uma posição intermediária, povoada por instabilidades e turbulências que alimentam o risco de os sujeitos caírem na situação de desfiliação.

Além de insegurança, incerteza e exposição a riscos, vulnerabilidade, em sua faceta social, traz consigo a ideia de desvantagem e desigualdade no que se refere ao acesso às condições de promoção e garantia de cidadania. Nesse sentido, o termo vulnerabilidade recebeu uma caracterização social. A noção de vulnerabilidade social ganhou estatuto de conceito cuja definição, complexa e multifacetada, vem sendo estruturada a partir de diversos olhares. Para não nos perdermos na multiplicidade de sentidos que podem ser atribuídos a esse termo, é preciso ter em mente, pelo menos, duas perguntas: qual é a abrangência de uma situação de vulnerabilidade social? Quais são as consequências de se atribuir a condição de vulnerabilidade social a um jovem?

A condição de vulnerabilidade social pode ser definida a partir da falta ou da não condição de acesso a bens materiais e de serviços que possam suprir aquilo cuja ausência torna o indivíduo vulnerável (Ayres et al., 1999). Neste momento, é importante notar que a palavra vulnerável remete à possibilidade de o sujeito sofrer algum tipo de prejuízo. Ao definirem a condição de vulnerabilidade social como uma situação cujos recursos e habilidades são insuficientes e inadequados para obter acesso às oportunidades oferecidas 


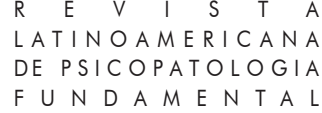

pela sociedade, Abramovey et al. (2002) incluem, de forma clara, restrições que impossibilitam a movimentação entre as estruturas econômicas e sociais. Deste modo, é possível entender que um suposto prejuízo não está por vir, mas que já existem perdas e danos instaurados, por exemplo, pelo emprego precário e pela insuficiência de renda. Isto, por si só, já é prejudicial.

Deve ficar claro que o prejuízo não se restringe à categoria econômica. Ao lado desta, faz-se necessário incluir vicissitudes e idiossincrasias que vão além dos atributos de renda, tais como questões ligadas a preconceitos, à falta de escolaridade, à violência doméstica e urbana. Essas questões, além de determinarem algum tipo de desigualdade em termos de inserção na sociedade, colocam em cena uma gama de marcas identitárias produzidas pela condição de vulnerabilidade social: ser pobre, ser negro, ser da periferia, ser analfabeto elou ser morador de comunidade. Sabemos que tais rótulos podem determinar lugares sociais que estigmatizam e produzem a inscrição de marcas identitárias cujas repercussões ultrapassam o plano econômico e social, e acabam causando efeitos na dimensão subjetiva. Esses efeitos serão abordados a seguir.

\section{Marcas da vulnerabilidade social e expressões do sofrimento psíquico}

Para iniciar uma discussão acerca dos efeitos produzidos pela marcas identitárias inscritas pela condição de vulnerabilidade social, torna-se necessário, antes de mais nada, partir da ideia de que a dimensão subjetiva não pode ser desvinculada do ambiente no qual o sujeito encontra-se inserido. Winnicott (1945/2000a) fornece um sentido amplo à noção de ambiente, definindo-a, sobretudo, a partir do cuidado exercido pela abrangência do entorno do qual o sujeito faz parte. Dessa forma, o termo ambiente pode ser entendido como sinônimo de uma gama de situações que abrangem desde o cuidado exercido pela mãe até a garantia e manutenção de direitos do cidadão e deveres por parte do Estado, passando pelo papel simbólico exercido por instituições como as famílias, as comunidades, os grupos eletivos, a escola e as igrejas.

O ambiente precário no qual os sujeitos em condição de vulnerabilidade social vivem deve ser entendido como um meio que não provê as necessidades fundamentais, tais como moradia, educação e cuidado. Faltas de cuidado e de acolhimento fornecidas pelo ambiente podem ser nomeadas, segundo Winnicott (1952/2000b), falhas ambientais. Esse tipo de não 


\section{ARTIGOS}

provimento ambiental, ou digamos assim, de provimento ambiental falho, incide de modo fortemente negativo no reconhecimento das potencialidades do sujeito e no uso que poderá ser feito delas no enfrentamento das adversidades da vida.

Recentemente, alguns autores vêm usando o termo sofrimento social (Carreteiro, 2003; Renault, 2008) para fazer referência a diferentes tipos de sofrimento causados por desigualdades sociais que desvalorizam e humilham, fazendo com que os sujeitos se sintam envergonhados (Sawaia, 1999). O que está em pauta são situações sociais que depreciam a importância dos códigos sociais e culturais de determinada comunidade ou grupo, invalidando-os e, consequentemente, negando o lugar social do sujeito (Czermak, 2016). Ao apresentar três dimensões do sofrimento social (vergonha, humilhação e falta de reconhecimento), Carreteiro (2003) defende a ideia de que esse tipo de sofrimento é invisível e silencioso: "ele se inscreve no interior das subjetividades sem, no entanto, ser compartilhado coletivamente" (p. 57). Quando não há o reconhecimento de potencialidades subjetivas, ou, dito de outro modo, quando o reconhecimento do sujeito existe pela via da negatividade, vão sendo produzidas marcas - oriundas da invalidação, da depreciação e da desqualificação - constitutivas da própria imagem, fazendo com que os sujeitos obtenham reconhecimento simbólico a partir da condição de inadequação.

É justamente a partir dessa lógica que Honneth (2003) define o que nomeou recusa de reconhecimento. Para esse autor, o reconhecimento pode ser considerado uma necessidade vital, pois todo sujeito, para desenvolver suas potencialidades, precisa ser visto, ouvido e respeitado no que diz respeito, sobretudo, à esfera do amor, à esfera do direito e à esfera da solidariedade. $\mathrm{O}$ não reconhecimento nessas três esferas pode impedir, respectivamente, o estabelecimento da autoconfiança, do autorrespeito e da autoestima, podendo gerar, até mesmo, morte psíquica, morte social e humilhação. Diante disso, é possível conceber a ideia de que um reconhecimento recusado pode ser entendido como uma situação em que o ambiente falha em sua função de prover as necessidades fundamentais. Nesse sentido, tanto o não reconhecimento, quanto o reconhecimento estigmatizante, pelo negativo, ferem, deixando marcas que impedem o sujeito de apreender suas próprias potencialidades.

Ao analisar os conceitos ferenczianos a partir de uma ótica política, Gondar (2012) empreende uma tentativa de estender as noções de trauma e desmentido para o campo social. Nesse contexto, o desmentido é articulado à 


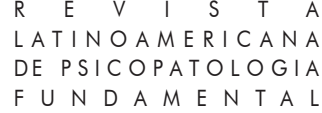

noção de recusa de reconhecimento em Honneth, podendo então ser entendido como o avesso do reconhecimento. Para Ferenczi, o desmentido está diretamente relacionado à incapacidade do ambiente de validar, dar crédito e de funcionar como mediador de sentido para as experiências do sujeito. Tal falha contém em si potência traumática. Com a intenção de fundamentar o caráter traumático do desmentido, em 1933, no artigo "Confusão de língua entre os adultos e a criança", Ferenczi adota o mecanismo de sedução da criança pelo adulto como paradigma. Nessa situação, mais do que o ato em si, o que merece destaque é a falta de crédito dada ao sofrimento da criança violada: a não validação da violência sofrida, por parte do adulto, torna o trauma patogênico. Desta forma, o que traumatiza é a falha do provimento de uma das necessidades vitais do sujeito postuladas por Honneth: a de reconhecimento. Em suma, é possível afirmar que o desmentido do sofrimento pelo ambiente que torna o trauma patogênico, em Ferenczi, pode ser articulado à noção de recusa de reconhecimento em Honneth.

Também interessado em discutir o que torna o trauma patogênico, Khan (1963/1977), influenciado pelas contribuições de Freud (1920/1990) e Winnicott (1952/2000b; 1967/1975), formula a noção de trauma cumulativo.

118 Em sua concepção, o trauma só se constitui como patogênico a partir do somatório de situações traumáticas devido às falhas repetidas do ambiente em sua função de prover as necessidades do sujeito. Assim, é possível associar a vivência de uma situação traumática com a abertura de uma fenda que deixa uma marca. A partir de tal lógica, o caráter patogênico não se efetua pelos acontecimentos no momento de sua ocorrência, isto é, durante a abertura das fendas, mas se instala pela repetição e pelo acúmulo de pequenas fissuras, silenciosa e invisivelmente. O trauma cumulativo resulta do somatório de fendas inscritas e acumuladas ao longo do tempo, o que significa que o fator traumático só pode ser percebido como tal após a instalação dos efeitos patogênicos das rachaduras acumuladas (Khan, 1963/1977).

Não é difícil perceber a articulação entre a noção de trauma cumulativo (Khan, 1963/1977) e as expressões do sofrimento psíquico produzidas pelo desmentido (Ferenczi, 1933/2011). Ao longo do tempo o sujeito gradualmente vai acumulando feridas que foram impressas pelo não reconhecimento e pelo desmentido dos efeitos psíquicos de situações de vulnerabilidade social. É assim que gradualmente são abertas feridas. Nesse sentido, o potencial do sujeito não é reconhecido como positivo, mas sim, a partir da negatividade. $\mathrm{O}$ acúmulo das feridas se configura como patogênico quando a humilhação, a vergonha e a falta de reconhecimento se instauram como marcas identitárias. 


\section{ARTIGOS}

Esse estado de coisas compromete a capacidade do sujeito de se relacionar com o ambiente, abalando a possibilidade de reconhecer suas potencialidades e de encontrar apoio nas próprias percepções.

Parece, então, que é possível afirmar que as teorias de Ferenczi e Khan, na sua articulação com as ideias de Honneth, fornecem elementos para o entendimento de que o caráter traumático adquirido pelos repetitivos e silenciosos golpes provocados pelo desmentido, interferem no reconhecimento das potencialidades do sujeito. Quando essa condição passa a constituir a marca do lugar ocupado pelo sujeito na trama social, torna-se necessário reconhecê-la. Escutar, dar crédito, validar, em suma, reconhecer os efeitos psíquicos de situações de vulnerabilidade social permite dar visibilidade ao sofrimento. Este é um primeiro passo indispensável para qualquer estratégia de mudança de sua posição no mundo.

\section{Escutando jovens em situação de vulnerabilidade social: papo reto e tá na roda}

Com o intuito de construir espaços de escuta para jovens em situação de vulnerabilidade social, foi iniciada, em março de 2015, uma pesquisaintervenção com moradores de comunidades do Rio de Janeiro, com idades entre 15 e 24 anos, matriculados na ONG Galpão Aplauso.

A primeira estratégia de intervenção utilizada foi a observação participante. Com o intuito de estar entre para conhecer, foi possível constatar, durante as oficinas artísticas ministradas como etapa do curso de capacitação profissional, a emergência de temas que apontavam para a necessidade dos jovens de serem mais bem compreendidos e assimilados. Por um lado, foram relatadas situações em que a condição de vulnerabilidade social aparecia como temática central, tais como desabamento da casa, morte do pai, tentativa de estupro, trabalho para o tráfico etc. Por outro, apareceram questões relativas à sexualidade, à dificuldade de diálogo em casa e à ansiedade para chegar a uma escolha profissional.

O material coletado possibilitou a elaboração do projeto-piloto "Escutando jovens em busca de um futuro profissional: intervenções psicanalíticas em um contexto educativo não formal". Essa primeira abordagem foi estruturada com o intuito de oferecer aos jovens um espaço de escuta, tanto individual quanto em grupo. Dessa forma, duas modalidades de intervenção foram colocadas em prática: papo reto e tá na roda. 


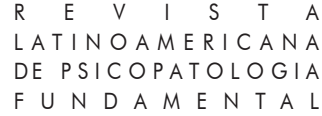

A modalidade de intervenção individual, papo reto, foi elaborada com a finalidade de proporcionar aos alunos um espaço de escuta pontual. A ideia para a construção desse dispositivo encontra-se baseada na proposta das "consultas terapêuticas" (Winnicott, 1971/1984), concebidas como um uso da psicanálise no campo da psiquiatria infantil. O objetivo principal dessa modalidade de intervenção consiste em explorar integralmente a primeira entrevista com a premissa de que, durante a investigação da demanda subjetiva apresentada, uma ação terapêutica possa ser realizada. A escuta e o acolhimento do material emergente no primeiro encontro podem ser terapêuticos na medida em que conduzem à elaboração de uma questão ou de um sofrimento.

Durante o ano de 2015, 16 jovens foram escutados individualmente. $\mathrm{O}$ trabalho feito foi o de transformar o pedido de ajuda, expresso, na maioria das vezes, sob a forma de uma pergunta direta ou a solicitação de conselho, em uma reflexão acerca de si mesmo. Um dos jovens procurou a psicanalista com as seguintes questões: "Gosto de garotas e de garotos. Já namorei com os dois. Será que tenho dupla identidade sexual? Não sei o que sou”. A partir de tais perguntas foi possível dar início à reflexão acerca da diferença existente entre preferências sexuais, identidade e caráter de cada um. Logo no início de uma outra entrevista um jovem diz o seguinte: "Sou muito nervoso, estou como medo de perder meus amigos. Como posso me controlar?”. Ao longo do encontro foi possível localizar que, por trás de tal pedido, havia a fantasia de ter sido o responsável pela separação dos pais. Um outro jovem, solicita "uma conversa" e expressa a seguinte angústia: "Estou com medo de morrer quando fizer 18 anos, o que faço?". O pedido de conselho revela a dificuldade de conversar com a mãe, o medo de se "perder para o tráfico" e acabar na cadeia como os irmãos. Ao final da entrevista foi possível deslocar a relação estabelecida entre o medo da morte e a chegada da maioridade para uma reflexão sobre o início da vida adulta, a responsabilidade pelas escolhas e a falta de ter com quem conversar sobre medos e angústias. Estes exemplos refletem como se deram os primeiros encontros estabelecidos com os jovens: pontuais, maleáveis e prontos para serem desdobrados.

Tendo como base o material obtido na modalidade de intervenção descrita acima, e o fato de os pesquisadores terem se tornado conhecidos entre os jovens, foi proposta uma forma coletiva de trabalho nomeada tá na roda. Esse dispositivo foi estruturado com o intuito de favorecer a circulação da palavra para promover discussões e reflexões acerca de temas e questões levantadas pelos participantes. 


\section{ARTIGOS}

O tá na roda foi construído baseado na proposta das "conversações" (Miller, 1997; 2003), atualmente, adotadas pelo Centre Interdisciplinaire sur l'Enfant (CIEN) como instrumento de pesquisas realizadas na interface entre psicanálise e educação (Lacadeé, 1999-2000). O ponto de partida desse dispositivo consiste em possibilitar uma espécie de associação livre coletivizada - fundamentada na premissa de que um significante remete a outro significante - com o objetivo de permitir que cada sujeito envolvido possa tomar a palavra e agir inspirado pelo discurso dos outros. A ideia principal é que a construção de uma associação livre coletivizada acaba atingindo o singular, provocando um efeito de subjetivação que, por sua vez, proporciona a elaboração de uma narrativa em primeira pessoa e, com isso, a construção de um saber sobre si: "quando as coisas vão bem, os significantes dos outros me dão ideias, me ajudam e, finalmente, resulta — às vezes - algo novo, um ângulo novo e perspectivas inéditas" (Miller, 2003, p. 15-16).

Como parte do projeto-piloto, foram realizados quatro encontros com frequência semanal e uma hora de duração. Além dos oito jovens matriculados em uma oficina artística oferecida pela instituição, participaram duas psicanalistas, um aluno de iniciação científica e o professor daquela oficina. Durante a realização da atividade proposta, o transitivismo e o uso do outro como espelho funcionaram como meios de estabelecer contato com as marcas identitárias inscritas pela condição de vulnerabilidade social e com as particularidades características do momento de transição vivido pelos jovens. Nesse contexto, observamos que, na maioria das vezes, os participantes falavam de si falando dos outros ou até mesmo pelos outros. Dessa forma, o oferecimento de um espaço em que a palavra circula possibilitou o trânsito de identificações: projetando-se nos outros, os jovens encontraram possibilidades tanto de se identificarem quanto de se diferenciarem.

Logo no primeiro dia do uso do dispositivo proposto, foi possível perceber que um participante respondia pelo outro diversas vezes e também que alguns jovens tomavam a palavra para falar em nome de seus colegas. Essa dinâmica, geralmente, era acompanhada por uma espécie de "zoação", uma forma de zombar amistosamente: "M. veio para cá para a mãe dela parar de encher o saco"; " $W$. se acha! Tem talento, mas se acha". Participando do tipo de comunicação estabelecida, um jovem, após ter estabelecido pequenos confrontos com seus colegas, acabou reconhecendo que gostava de implicar com os outros. A comunicação desse reconhecimento permitiu que, aos poucos, os outros participantes fossem se envolvendo e se comprometendo com a proposta de conversar livremente em grupo, sem 


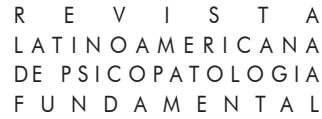

amarras e sem um roteiro ou conteúdo preestabelecidos. Assim, a forma amistosa de zombar dos outros permitiu que os jovens pudessem se ver a partir do olhar do outro e, por sua vez, devolver esse olhar. A dinâmica estabelecida abriu o caminho para o uso de projeção como uma forma de comunicação: projetando-me no outro, encontro minha imagem. Tal tipo de identificação possibilitou a construção de uma narrativa coletiva acerca dos desejos e das questões que envolviam a transitoriedade atravessada e a situação de vulnerabilidade social experimentadas pelos jovens.

É interessante notar como essa mesma dinâmica permite que a conversa transite de tópicos mais objetivos para temas mais subjetivos e íntimos. Em um dos encontros, por exemplo, foi possível, a partir da iniciativa de uma das jovens, iniciar conversando sobre desigualdade e terminar conversando sobre sentimento. Assim, a seguinte trilha associativa foi estabelecida: desigualdade social — políticos — injustiça — promessas — desilusão amor — amor de mãe, de irmão — amar - se apaixonar — amor próprio - cuidar de si - não se amar - masoquismo - morrer pelo outro - tipos e intensidades de amor - amor construido - amor de mãe - falta de amor de mãe - incapacidade de amar - Renato Russo - Cássia Eller - drogas

122 - bissexualidade - poesia - arte - comunicação - sentimento. No final desse encontro, um participante disse que havia gostado da atividade devido ao fato de que "falar aqui dentro não é igual a falar lá fora: aqui a gente pode falar, ouvir e respeitar".

A fala desse participante permite afirmar que a construção de espaços de escuta para jovens em situação de vulnerabilidade social pode efetivamente instaurar lugares que permitem testemunhar, reconhecer, legitimar e promover a circulação da palavra em sua multiplicidade de sentidos e em sua plasticidade criadora. Escutar significa colocar em prática formas de se contrapor à naturalização e à invisibilidade que caracterizam situações que muitas vezes foram silenciadas. Sendo assim, ouvir, deixar falar, e possibilitar a afetação a partir de narrativas do cotidiano ou de gestos invalidados pela falta de reconhecimento pode conduzir o sujeito ao encontro de suas potencialidades.

\section{Considerações finais: entre a vulnerabilidade e o reconhecimento}

Muitas vezes, as marcas inscritas pela condição de vulnerabilidade social passam a se constituir como formas, naturalizadas pela sociedade, de 


\section{ARTIGOS}

definir e caracterizar os atributos e o laços do sujeito com o ambiente. De acordo com o que foi apresentado, é possível afirmar que o caráter traumático, produtor de sofrimento, resulta da exposição a situações em que o golpe subjetivo desferido pelo desmentido é repetido ao longo do tempo. A falta de reconhecimento da condição de vulnerabilidade dos sujeitos por parte do ambiente contribui para a banalização da injúria sofrida.

Em sua teoria a respeito da luta pelo reconhecimento, Honneth (2003) parte da ideia de que o reconhecimento mútuo é não só uma necessidade vital do humano, como também a forma crucial de estabelecimento de um status de unidade para o eu. Na visada desse autor, o reconhecimento do eu só pode ser efetuado a partir do olhar do ambiente. É a partir da relação estabelecida com a alteridade que o sujeito pode encontrar suas potencialidades. Seguindo essa lógica, é possível entender que quando o sujeito não encontra o reconhecimento de si no ambiente, ele é silenciado: suas potencialidades tornam-se inaudíveis e invisíveis.

A invalidação perceptiva e afetiva da condição de vulnerabilidade do sujeito coloca em cena o ambiente em sua faceta traumática. $\mathrm{O}$ reconhecimento das adversidades do ambiente pode permitir ao sujeito tomar posse de sua condição de vulnerabilidade e, consequentemente, das percepções que foram desmentidas e, portanto, silenciadas. A partir da circulação da palavra, entre a vulnerabilidade e o reconhecimento, é possível identificar, nomear e dar sentido à precariedade que sobreviveu à tentativa de anulação e apagamento imposta pelo desmentido.

Sendo assim, é somente a partir da constatação da condição de vulnerabilidade que o sujeito pode reconhecer a singularidade de suas potencialidades. É nessa direção que a construção de espaços de escuta pretende operar: ouvir, deixar falar, identificar, nomear e reconhecer são ações que contribuem para a adoção de novos sentidos e significados para posições cristalizadas pela condição de vulnerabilidade social.

Agradecimentos: Gostaria de agradecer a ONG Galpão Aplauso, sobretudo a Ivonette Albuquerque, Ana Melo e Claudia Esquerdo, pela cooperação estabelecida; às colegas do Núcleo Comunitário da Clínica Social do Círculo Psicanalítico do Rio de Janeiro (CPRJ) - Claudia Garcia, Fania Izhaki, Luciana Lenz Cesar e Maria Regina Maciel - pela parceria de trabalho, pela leitura e pelos comentários do texto e, finalmente, ao aluno Victor Guagliardi pelo registro das observações realizadas. 


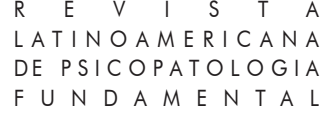

\section{Referências}

Abramovey, M. et al. (2002). Juventude, violência e vulnerabilidade social na América Latina: desafios para políticas públicas. Brasília, DF: Unesco.

Ayres, J. R. et al. (1999). Vulnerabilidade e prevenção em tempo de AIDS. In R. Parker et al. Sexualidade pelo avesso: direitos, identidades e poder. São Paulo, SP: Editora 34.

Castel, R. (1999, jan./dez.). A dinâmica dos processos de marginalização: da vulnerabilidade à "desfiliação". Caderno CRH, 26/27, 19-40. Recuperado de http://www.cadernocrh.ufba.br/viewarticle.php?id=193.

Carreteiro, T. C. (2003). Sofrimentos sociais em debate. Psicologia USP, 14(3), $57-$ 72.

Czermak, S. (2016). Arte de fazer com os sons: escuta psicanalítica da prática orquestral. Tese de Doutorado em Psicologia Clínica, Departamento de Psicologia. PUC-Rio, Rio de Janeiro.

Ferenczi, S. (2011). Confusão de língua entre adultos e criança. In Obras completas de Sándor Ferenczi (Vol. IV). São Paulo, SP: Martins Fontes. (Trabalho original publicado em1933).

124 Freud, S. (1990). Além do princípio do prazer. In Edição Standard Brasileira das Obras Psicológicas Completas de Sigmund Freud (Vol. XVII, pp. 13-88). Rio de Janeiro, RJ: Imago. (Trabalho original publicado em 1920).

Gondar, J. (2012, jul./dez.). Ferenczi como pensador político. Cadernos de Psicanálise, 34(27), 193-210.

Honneth, A. (2003). Luta por reconhecimento: gramática moral dos conflitos sociais. São Paulo, SP: Editora 34.

Khan, M. (1977). O conceito de trauma cumulativo. In Psicanálise: teoria, técnica e casos clínicos. Rio de Janeiro, RJ: Francisco Alves. (Trabalho original publicado em 1963).

Klautau, P., \& Faissol, K. (2015 nov., 2016 abr.). Do Nebenmensch ao Unheimelich: a presença da alteridade no processo de constituição da subjetividade. Revista aSEPHallus de Orientação Lacaniana, 11(21), 66-76.

Lacadeé, P. (1999-2000). De la norme de la conversation au détail de la conversation. In P. Lacadeé, \& F. Monier (Orgs.), Le Pari de la Conversation. Institut du Champs Freudien: CIEN (Centre Interdisciplinaire sur l'Enfant. Paris. (brochuere)

Miller, J.-A. Os casos raros, inclassificáveis da clínica psicanalítica. A conversação de Arcachon. São Paulo, SP: Biblioteca Freudiana Brasileira, 1997.

Miller, J.-A. La Pareja y el Amor: conversaciones clinicas en Barcelona. Barcelona: Eólia, 2003. 


\section{ARTIGOS}

Renault, E. (2008). Souffrances socialles: sociologie, psychologie et politique. Paris: Le Découverte.

Sawaia, B. (1999). Identidade - uma ideologia separatista. In B. Sawaia (Org.), As artimanhas da exclusão. Petrópolis, RJ: Vozes.

Winnicott, D. W. (1975). A localização da experiência cultural. In O brincar e a realidade. Rio de Janeiro, RJ: Imago. (Trabalho original publicado em 1967).

Winnicott, D. W. (1984). Consultas terapêuticas em psiquiatria infantil. Rio de Janeiro, RJ: Imago. (Trabalho original publicado em 1971).

Winnicott, D. W. (2000a). O desenvolvimento emocional infantil. In Da pediatria à psicanálise. Rio de Janeiro, RJ: Imago. (Trabalho original publicado em 1945).

Winnicott, D. W. (2000b). Psicoses e cuidados maternos. In Da pediatria à psicanálise. Rio de Janeiro, RJ: Imago. (Trabalho original publicado em 1952).

\section{Resumos}

(The psychoanalytic method and its extensions: listening to young people in situation of social vulnerability)

This paper discusses efforts to establish places for listening to young people in social vulnerability situations. To this end, two types of intervention were created; they were developed at individual and group scope, and respectively named "papo reto" (straightforward conversation) and "tá na roda" (let's share). Proposals such as these allow for progress towards the establishment of tools that are an alternative to classical settings, extending the psychoanalytic method's contributions to health, education and culture.

Keywords: Psychoanalysis in extension, social vulnerability, trauma, identification

(La méthode psychanalytique et son développement hors du champ classique: l'écoute des jeunes en situation de vulnérabilité sociale)

Cet article présente le travail de construction d'espaces d'écoute pour des jeunes en situation de vulnérabilité sociale. Pour ce faire, deux modalités d'intervention ont été mis en place, la première dans le domaine individuel et la deuxième dans celui du groupe, nommées respectivement " papo reto » et " tá na roda ». Ces propositions ont pour objectif d'établir une collaboration visant à construire des dispositifs alternatifs au setting classique afin de développer la contribution de la méthode psychanalytique à la santé, à l'éducation et à la culture.

Mots clés: Psychanalyse en développement, vulnérabilité sociale, trauma, identification 
(El método psicoanalítico y sus extensiones: escuchando a jóvenes en situación de vulnerabilidad social)

Este artículo propone una discusión sobre el trabajo de construcción de espacios de escucha para jóvenes en situación de vulnerabilidad social. Para ello, fueron configuradas dos modalidades de intervención, desarrolladas en los ámbitos individual y grupal, denominadas, respectivamente, "papo reto" (charla directa) y "tá na roda" (discutiendo juntos). Propuestas como estas permiten el avance en dirección a estrategias de construcción de dispositivos alternativos al setting clásico, extendiendo las contribuciones del método psicoanalítico a la salud, la educación y la cultura.

Palabras clave: Psicoanálisis en extensión, vulnerabilidad social, trauma, identificación

(Die psychoanalytische Methode und ihre Erweiterungen: Jungen Leuten in sozialer Vulnerabilität zuhören)

Der vorliegende Artikel diskutiert die Bildung von Bereichen des Zuhörens für junge Menschen, die sozialer Vulnerabilität ausgesetzt sind. Dazu wurden zwei Interventionsarten gewählt, die im Bereich des Individuums und der Gruppe entwickelt wurden und jeweils „,Klartextreden “ und „,in die Runde bringen “ genannt wurden. Vorschläge dieser Art zielen darauf hin, neben dem klassischen Setting Alternativen zu setzen und die Beiträge der psychoanalytischen Methode zum Gesundheits-, Erziehungs- und Kulturwesen zu erweitern.

Schlüsselwörter: Erweiternde Psychoanalyse, SozialeVulnerabilität, Trauma, Identifikation

（精神分析方法及其扩展：倾听处于社会弱势的年青人）

本文旨在讨论针对处于社会弱势的年青人的心理倾听工作。为此, 我们设置了两种类型的干预, 一是个别干预, 二是团体性干预, 分别命 名为 “直诉” (papo reto) 和 “轮转” (tá na roda)。建立这种心理聆 听空间有助于突破传统的青少年教育理念, 促进年青人精神健康, 保护 青少年在教育和文化方面的顺利发展。

关键词：精神分析的扩展；社会脆弱性；创伤；认同。

Citação/Citation: Klautau, P. (2017, março). O método psicanalítico e suas extensões: escutando jovens em situação de vulnerabilidade social. Revista Latinoamericana de Psicopatologia Fundamental, 20(1), 113-127.

Editores do artigo/Editors: Profa. Dra. Ana Maria Rudge e Profa. Dra. Sonia Leite 


\section{ARTIGOS}

Recebido/Received: 9.9.2016/ 9.9.2016 Aceito/Accepted: 10.11.2016 / 11.10.2016

Copyright: (C) 2009 Associação Universitária de Pesquisa em Psicopatologia Fundamental/ University Association for Research in Fundamental Psychopathology. Este é um artigo de livre acesso, que permite uso irrestrito, distribuição e reprodução em qualquer meio, desde que o autor e a fonte sejam citados / This is an open-access article, which permits unrestricted use, distribution, and reproduction in any medium, provided the original authors and sources are credited.

Financiamento/Funding: Pesquisa financiada pela Fundação de Amparo à Pesquisa do Rio de Janeiro - Faperj / The research was funded by Fundação de Amparo à Pesquisa do Rio de Janeiro - Faperj

Conflito de interesses/Conflict of interest: A autora declara que não há conflito de interesses / The author has no conflict of interest to declare.

Perla Klautau

Psicanalista; Membro Efetivo do Círculo Psicanalítico do Rio de Janeiro - CPRJ (Rio de Janeiro, RJ, Br); Professora dos Programas de Mestrado Profissional e Doutorado em Psicanálise, Saúde e Sociedade da Universidade Veiga de Almeida - UVA (Rio de Janeiro, $\mathrm{RJ}, \mathrm{Br})$.

Rua Ibituruna, 108 - casa 3 - Maracanã

20271-901 Rio de Janeiro, RJ, Br

pklautau@uol.com.br

This is an open-access article, which permits unrestricted use, distribution, and reproduction in any medium for non-commercial purposes provided the original authors and sources are credited.

Rev. Latinoam. Psicopat. Fund., São Paulo, 20(1), 113-127, mar. 2017 